\title{
Pflanzliches Antidementivum
}

\section{Mehr Power für die neuronalen Kraftwerke}

\section{In der Entstehung der Alzheimer- demenz rücken die neuronalen Mitochondrien immer stärker in den Fokus der Forschung.}

- Bereits im Rahmen des physiologischen Alterungsprozesses nimmt der oxidative Stress in den Mitochondrien zu: In diesen sammeln sich vermehrt reaktive Sauerstoffspezies (ROS) an. Nach aktuellen Studiendaten liegt bei Alzheimerpatienten ein Enzymmangel in den Mitochondrien vor, der den Überschuss an ROS weiter verstärkt. Die Folgen sind eine weiter verminderte Produktion des energiereichen Adenosintrophosphats (ATP), ein Funktionsverlust und schließlich die Apoptose der Neu-

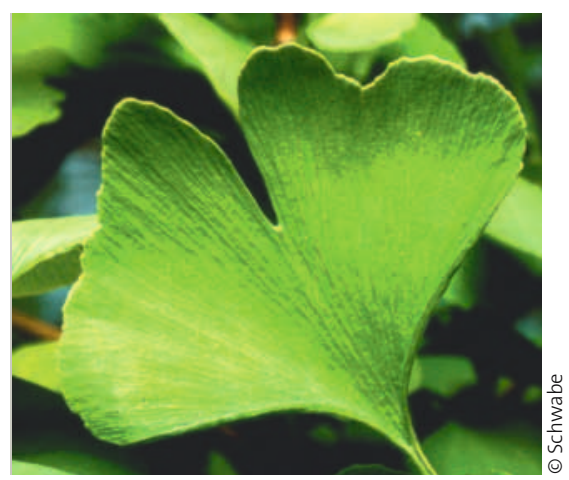

Ginkgo biloba: Vielfältige Wirkungen auf das ZNS. ronen (Reddy $\mathrm{PH}$ et al. Trends Mol Med 2008).

\section{Schutz der Mitochondrien}

Ginkgoflavonglykoside (z. B. Quercetin) sowie Terpenlactone (Ginkgolide und Bilobalid), die pharmazeutisch wirksamen Inhaltsstoffe in Ginkgoblättern, haben vielfältige Wirkungen auf das ZNS: Sie unterstützen die Bildung dendritischer Fortsätze der Neuronen im Hippocampus, aber auch die Ausbildung neuer Synapsen, etwa wenn diese durch Amyloid-beta geschädigt sind (Tchantchou et al. J Alzheimer's Dis 2009; Luo $Y$ et al. PNAS 2002). Sie fördern den Brain-derived neurotrophic Faktor (BDNF), einen Nervenwachstumsfaktor, und wirken als Radikalfänger. Dabei schützen und stabilisieren sie die neuronalen Mitochondrien und damit die Energiebereitstellung und besonders vulnerable Funktionsabläufe der Signalübertragung und Zellbiologie.

Präklinische Studien zeigten, dass ein standardisierter Ginkgo-biloba-Extrakt die Mitochondrien schützt: EGb $761^{\circledR}$ konnte am Modell der PC-12-Zellen die mitochondriale ATP-Ausbeute unter oxidativem Stress verbessern (Eckert A et al, Pharmacopsychiatry 2003).

\section{Besser vernetzt mit Ginkgo}

Um das mentale Potenzial voll ausschöpfen zu können, ist weniger die Anzahl der Nervenzellen als vielmehr ihre Vernetzung von Bedeutung. Speziell im Hippocampus, dem „Tor zum Gedächtnis“ ist eine Abnahme der Zellverbindungen fatal: Sie wird mit einer nachlassenden mentalen Leistungsfähigkeit quittiert. Eine internationale
Forschungsgruppe um Prof. Yuan Luo, Baltimore (Maryland/USA), konnte zeigen, dass Ginkgo-Extrakt EGb $761^{\circ}$ die Hippocampuszellen dazu anregen kann, neue Verbindungen zwischen einzelnen Zellnetzwerken zu schaffen und diese zu verdichten bzw. weiter auszubauen.

KW .

- Luo Y et al.J Alzheimer Dis 2009;18:787-98 